\title{
Seleção recorrente recíproca na obtenção de híbridos interpopulacionais de milho-pipoca
}

\author{
Vinícius Ribeiro Faria ${ }^{(1)}$, José Marcelo Soriano Viana( ${ }^{(1)}$, Fábio Moreira Sobreira ${ }^{(1)}$ e Admilson Costa e Silva ${ }^{(1)}$ \\ (1)Universidade Federal de Viçosa, Departamento de Biologia Geral, Avenida P.H. Rolfs, s/nº, Campus Universitário, CEP $36570-000$ Viçosa, \\ MG. E-mail: virfaria@yahoo.com.br, jmsviana@ufv.com.br, fabiomsobreira@yahoo.com.br, admilsoncostaesilva@yahoo.com.br
}

Resumo - O objetivo deste trabalho foi avaliar a eficiência da seleção recorrente recíproca em produzir híbridos de milho-pipoca (Zea mays) de progênies endógamas superiores, com famílias de irmãos-completos. O programa de seleção recorrente recíproca envolveu as populações de milho-pipoca 'Viçosa' e 'Beija-Flor'. Os testes dos híbridos $\mathrm{S}_{0} \mathrm{xS}_{0}$ e $\mathrm{S}_{1} \mathrm{xS}_{1}$ foram conduzidos em delineamento látice, nos anos agrícolas de 2002/2003 e 2004/2005. Os dois ensaios incluíram testemunhas comerciais comuns, que permitiram a comparação do desempenho dos híbridos. Analisaram-se a produtividade e a capacidade de expansão. Foram preditos ganhos direto e indireto com a seleção em capacidade de expansão, avaliada em pipoqueira de ar quente e na pipocadora "Metric Weight Volume Tester". Os ganhos observados foram calculados para avaliar a eficiência da seleção recorrente recíproca. As análises de covariância mostraram variabilidade genotípica nas populações. Em relação às testemunhas comerciais, os híbridos $\mathrm{S}_{0} \mathrm{xS}_{0}$ foram inferiores em qualidade e equivalentes em produtividade, e os híbridos $\mathrm{S}_{1} \mathrm{x} \mathrm{S}_{1}$ foram equivalentes nas duas características. $\mathrm{O}$ ganho observado na capacidade de expansão foi substancial, cerca de $4 \mathrm{~mL} \mathrm{~g}^{-1}$, superior ao ganho predito $\left(2,8 \mathrm{~mL} \mathrm{~g}^{-1}\right)$. A redução observada na produtividade não foi relevante. Verificou-se redução na variabilidade genotípica quanto à capacidade de expansão. O método de seleção recorrente recíproca foi eficiente em produzir híbridos $\mathrm{S}_{1} \mathrm{xS}_{1}$ superiores aos $\mathrm{S}_{0} \mathrm{xS}_{0}$.

Termos para indexação: Zea mays, famílias de irmãos-completos interpopulacionais, ganho genético, método dos híbridos crípticos.

\section{Reciprocal recurrent selection to obtain interpopulation hybrids of popcorn}

Abstract-The objective of this work was to evaluate the efficiency of reciprocal recurrent selection in producing hybrids of popcorn (Zea mays) from superior endogamous progenies, with full-sibling families. The reciprocal recurrent selection program involved the 'Viçosa' and 'Beija-Flor' popcorn populations. Tests of the hybrids $\mathrm{S}_{0} \mathrm{XS}_{0}$ and $\mathrm{S}_{1} \mathrm{x} \mathrm{S}_{1}$ were carried out in lattice design, in the agricultural years 2002/2003 and 2004/2005. The two assays included common commercial controls, allowing for the comparison of the hybrids performance. Productivity and expansion capacity were evaluated. Direct and indirect gains with selection were predicted on expansion capacity assessed in hot air and Metric Weight Volume Tester popcorn makers. The gains observed were calculated to evaluate the efficiency of the reciprocal recurrent selection. The covariance analyses showed genotypic variability in the populations. When compared to the commercial controls, the $\mathrm{S}_{0} \mathrm{x} \mathrm{S}_{0}$ hybrids were inferior in quality and equivalent in productivity; and the $\mathrm{S}_{1} \mathrm{x} \mathrm{S}_{1}$ hybrids were equivalent in both traits. The observed gain in expansion capacity was substantial (around $4 \mathrm{~mL} \mathrm{~g}^{-1}$ ) and higher than the predicted gain $\left(2.8 \mathrm{~mL} \mathrm{~g}^{-1}\right)$. The reduction observed in productivity was not relevant. Reduction in the genotypic variability was verified for expansion capacity. The reciprocal recurrent selection method was efficient in producing $\mathrm{S}_{1} \times \mathrm{S}_{1}$ hybrids superior to $\mathrm{S}_{0} \mathrm{x} \mathrm{S}_{0}$ hybrids.

Index terms: Zea mays, interpopulation full-sib families, genetic gain, cryptic hybrids method.

\section{Introdução}

No Brasil, o milho-pipoca, apesar do elevado consumo, tem os processos de melhoramento e de produção pouco estudados. Há também escassez de informações oficiais sobre área semeada, produtividade, comercialização e importação (Pereira \& Amaral Júnior, 2001). Um dos maiores problemas enfrentados pelos produtores de milho-pipoca no país é o reduzido número de cultivares, que devem reunir boas características agronômicas com elevada qualidade. De acordo com Sawazaki et al. (2003), a reduzida disponibilidade de sementes híbridas de alta qualidade tem sido o principal responsável pelo crescimento da importação de grãos de milho-pipoca.

No melhoramento do milho-pipoca, podem ser aplicados os métodos utilizados no milho (Ziegler

Pesq. agropec. bras., Brasília, v.43, n.12, p.1749-1755, dez. 2008 
\& Ashman, 1994). Idealizado por Comstock et al. (1949), como um processo de melhoramento de duas populações e do híbrido entre elas, a seleção recorrente recíproca (SRR) com famílias de meios-irmãos paternos interpopulacionais e a recombinação de progênies $\mathrm{S}_{1}$ têm proporcionado, na maioria dos casos, resultados favoráveis - com milho e milho-pipoca -, em relação à produção, resistência a pragas e doenças, resistência ao acamamento, entre outros caracteres (Keeratinijakal \& Lamkey, 1993; Rademacher et al., 1999; Butron et al., 2000). A mesma afirmação pode ser feita em relação às modificações do método realizadas por Paterniani (1967), que sugeriu o uso de famílias de meios-irmãos maternos interpopulacionais e recombinação de progênies de meios-irmãos; e por Lonnquist \& Williams (1967) e Hallauer \& Eberhart (1970), que propuseram o emprego de progênies de irmãos-completos interpopulacionais e recombinação de famílias $S_{1}$, para o melhoramento das populações e do híbrido, e de famílias de irmãos-completos interpopulacionais endogâmicas, para a obtenção de híbridos de linhagens. Considera-se que o processo de SRR com irmãos-completos maximiza a seleção para capacidade específica de combinação, e favorece a obtenção de híbridos superiores de linhagens das populações envolvidas. Com esse propósito, Russell \& Eberhart (1975) sugeriram usar linhagem elite como testador, e Souza Júnior \& Pinto (2000) propõem obter híbridos do ciclo de seleção seguinte, ao mesmo tempo em que se realize a recombinação das progênies selecionadas do ciclo anterior. Com isso, o tempo de cada ciclo de seleção é reduzido, e as características originais do método são mantidas.

Com o milho, os primeiros resultados de SRR com irmãos-completos foram relatados por Hallauer (1973), que observou ganho em produção, de 10,1\%, no híbrido interpopulacional, depois de um ciclo de seleção envolvendo as populações 'Iowa Two Ear Synthetic' e 'Pioneer Ear Composite'. Hallauer (1984), ao trabalhar com famílias de irmãos-completos, também obteve resultados favoráveis, em sete ciclos de seleção, com as populações BS10 e BS11. A resposta direta na população híbrida foi de $2,1 \%$ por ciclo, para a produção, e as respostas correlacionadas nos parentais BS10 e BS11 foram de 2,7 e 2,4\%. Rademacher et al. (1999), ao utilizar SRR com irmãos-completos, nas populações BS21 e BS22, obtiveram aumento significativo em rendimento de grãos, atingindo $4,4 \%$ por ciclo. Esses autores observaram ainda que os ganhos realizados com seleção para rendimento de grãos representaram 25 a $50 \%$ do ganho predito. Ao utilizar famílias de irmãos-completos das populações IG-1 e IG-2, Santos et al. (2005) avaliaram o efeito de três ciclos de SRR sobre as médias, as variâncias genéticas e as correlações de várias características. Para produção de grãos foram observados ganhos de $4,07 \%$ por ciclo. Em relação à variância genética, foram observados pequenos decréscimos, insuficientes para limitar a melhoria da população e a obtenção de linhagens para formação de híbridos. Santos et al. (2007) avaliaram os efeitos de capacidade geral (CGC) e especifica (CEC) de combinação no processo de seleção recorrente recíproca modificada. Segundo esses autores, tanto a CGC quanto a CEC contribuem para a melhoria do híbrido da população, no entanto, a CEC tem maior contribuição para produtividade.

Pela grande diversidade de ambientes e de tecnologias de produção existentes no Brasil, é importante se ter variedades e híbridos de milho-pipoca adaptados a essas condições, de forma a garantir alta produtividade, aliada à elevada qualidade.

O objetivo deste trabalho foi avaliar a eficiência do método de seleção recorrente recíproca em produzir híbridos de milho-pipoca de progênies endógamas superiores, com famílias de irmãos-completos.

\section{Material e Métodos}

Foram utilizados híbridos $\mathrm{S}_{0} \mathrm{xS}_{0}$ e $\mathrm{S}_{1} \mathrm{x} \mathrm{S}_{1}$ do programa de seleção recorrente recíproca com as populações de milho-pipoca 'Viçosa' e 'Beija-Flor' ciclo 1. Os híbridos $\mathrm{S}_{0} \mathrm{xS}_{0}$ foram obtidos nas safras de 2000/2001 e de 2001/2002. Para obtenção de cada família de irmãos-completos interpopulacionais, foi escolhida uma planta de cada população, uma delas prolífica. A primeira espiga dessa planta foi autofecundada e a segunda polinizada pela planta da população recíproca, a qual também foi autofecundada. Os cruzamentos proporcionaram progênies de irmãos-completos interpopulacionais (híbridos $\mathrm{S}_{0} \mathrm{x} \mathrm{S}_{0}$ ) e as correspondentes famílias $\mathrm{S}_{1}$.

Os híbridos $\mathrm{S}_{1} \mathrm{xS}_{1}$ foram obtidos no ano agrícola de 2003/2004, e as famílias $S_{2}$ foram obtidas pela autofecundação das plantas cruzadas. Foram cruzados 45 pares de famílias $\mathrm{S}_{1}$ seguindo dois critérios: primeiramente, foram plantados os 20 pares que 
faziam parte de um trio completo - híbrido $\mathrm{S}_{0} \mathrm{xS}_{0}$, família $S_{1}$ de 'Viçosa' e família $S_{1}$ de 'Beija-Flor' -, independentemente do híbrido $\mathrm{S}_{0} \mathrm{x}_{0}$ ser selecionado ou não; os demais pares foram formados pelas 25 famílias $S_{1}$ de 'Viçosa' e pelas 25 famílias $S_{1}$ de 'Beija-Flor', cujas mães apresentaram maior valor de capacidade de expansão.

$\mathrm{O}$ teste dos híbridos $\mathrm{S}_{0} \mathrm{xS}_{0}$ foi conduzido na Universidade Estadual de Maringá, Maringá, PR, no ano agrícola de 2002/2003. Foram avaliadas 188 progênies de irmãos-completos. $\mathrm{O}$ delineamento utilizado foi látice $14 \times 14$ simples, e as testemunhas foram 'Ângela', 'Zélia', 'IAC 112', 'Viçosa', 'BeijaFlor' e as populações melhoradas 'Viçosa' ciclo 1, 'Beija-Flor' ciclo 1 e 'Beija-Flor' ciclo 2, obtidas por seleção entre e dentro de famílias de meios-irmãos. Cada parcela correspondeu a uma fileira de $5 \mathrm{~m}$, com 30 plantas. O espaçamento entre fileiras foi de $0,9 \mathrm{~m}$. As variáveis avaliadas foram produtividade $\mathrm{e}$ capacidade de expansão, avaliada em pipoqueira de ar quente, utilizando amostras de $30 \mathrm{~g}$, e na pipocadora Metric Weight Volume Tester (MWVT), com amostras de $250 \mathrm{~g}$.

A avaliação dos 136 híbridos $\mathrm{S}_{1} \mathrm{XS}_{1}$ foi realizada na estação experimental da Universidade Federal de Viçosa, em Ponte Nova, MG, na safra de 2004/2005. $\mathrm{O}$ delineamento utilizado foi látice $12 \times 12$, com três repetições. Cada parcela foi constituída de uma fileira de $5 \mathrm{~m}$, com 30 plantas. $\mathrm{O}$ espaçamento entre fileiras foi de $0,9 \mathrm{~m}$. Foram incluídas como testemunhas, as populações Ângela e Beija-Flor, ciclo 3, e os híbridos Zélia, IAC 112, Viçosa x Beija-Flor ciclo 1, Viçosa x (Amarelo x Rosa Claro), Beija-Flor x (Viçosa x Branco) e Viçosa $\mathrm{x}$ (Beija-Flor $\mathrm{x}$ Branco). As variáveis avaliadas foram produtividade e capacidade de expansão medida em pipoqueira de ar quente.

As análises de covariância foram realizadas utilizando o SAS, procedimento Mixed (Littell et al., 2006). O método de estimação foi quadrados mínimos ordinário, segundo o modelo:

$\mathrm{Y}_{\mathrm{ijk}}=\mu+\tau_{\mathrm{i}}+\mathrm{r}_{\mathrm{j}}+(\mathrm{b} / \mathrm{r})_{\mathrm{jk}}+\beta_{1} \mathrm{SF}_{\mathrm{i}}+\beta_{2} \mathrm{U}_{\mathrm{i}}+\mathrm{e}_{\mathrm{ijk}}$,

em que: $Y_{\mathrm{ijk}}$ é o valor fenotípico médio da parcela; $\mu$ é uma constante; $\tau_{\mathrm{i}}$ é o efeito de tratamento (efeito aleatório de híbrido e efeito fixo de testemunha); $\mathrm{r}_{\mathrm{j}}$ é o efeito de repetição (aleatório); $(\mathrm{b} / \mathrm{r})_{\mathrm{jk}}$ é o efeito de bloco dentro de repetição (aleatório); $\beta_{1}$ é o coeficiente de regressão para covariável estande final; $\mathrm{SF}_{\mathrm{i}}$ é o estande final da parcela; $\beta_{2}$ é o coeficiente de regressão para a covariável umidade; $U_{\mathrm{i}}$ é a umidade da parcela; e $\mathrm{e}_{\mathrm{ijk}}$ é o erro-experimental.

Nos cálculos dos ganhos esperados, foi considerada a seleção de $30 \%$ dos híbridos $\mathrm{S}_{0} \mathrm{xS}_{0}$ e $\mathrm{S}_{1} \mathrm{xS}_{1}$ e herdabilidade em sentido amplo, em média de famílias, dada por: $h^{2}=\hat{\sigma}_{\mathrm{g}}^{2} / \hat{\sigma}_{\mathrm{p}}^{2}($ Lynch \& Walsh, 1998),

em que $\hat{\sigma}_{\mathrm{g}}^{2}$ e $\hat{\sigma}_{\mathrm{p}}^{2}$ são os estimadores das variâncias genotípica e fenotípica entre médias de famílias, respectivamente.

Os ganhos diretos em capacidade de expansão foram preditos pela seguinte expressão: $\Delta \mathrm{M}=\mathrm{p} \times \mathrm{DS} \times \mathrm{h}^{2}$ (Lynch $\&$ Walsh, 1998), em que: $p$ é o controle parental $(\mathrm{p}=1)$ e DS o diferencial de seleção.

Os ganhos indiretos foram calculados pela expressão: $\Delta \mathrm{M}_{\mathrm{P}(\mathrm{CE})}=\mathrm{p}_{\times} \mathrm{DS}_{\mathrm{P}(\mathrm{CE})} \times \mathrm{h}_{\mathrm{p}}^{2}($ Lynch \& Walsh, 1998), em que: $\mathrm{DS}_{\mathrm{P}(\mathrm{CE})}$ é o diferencial de seleção indireta, obtido pela diferença entre a média de produtividade dos indivíduos selecionados com base na capacidade de expansão e a média geral; e $\mathrm{h}_{\mathrm{p}}^{2}$ é a herdabilidade em sentido amplo do caráter produtividade.

As correlações genotípicas foram calculadas pela expressão: $\hat{\rho}_{\mathrm{g}}=\operatorname{Cov}_{\mathrm{G}}(\mathrm{CE}, \mathrm{P}) / \sqrt{\hat{\sigma}_{\mathrm{g}_{\mathrm{CE}}}^{2} \hat{\sigma}_{\mathrm{g}_{\mathrm{p}}}^{2}}$ (Lynch \& Walsh, 1998), em que: $\operatorname{Cov}_{\mathrm{G}}$ é a covariância genotípica.

Para avaliar a eficiência da seleção recorrente recíproca, foram calculados os ganhos realizados, dados por: $\mathrm{GR}=\left(\mu_{\mathrm{S}_{1} \mathrm{~S}_{1}}-\mu_{\mathrm{S}_{0} \times \mathrm{S}_{0}}\right)-\left(\mu_{\mathrm{T}_{1}}-\mu_{\mathrm{T}_{0}}\right)$, em que: $\mu_{\mathrm{S}_{1} \mathrm{~S}_{1}} \mathrm{e}$ $\mu_{\mathrm{S}_{0} \mathrm{x} \mathrm{S}_{0}}$ são as médias dos híbridos $\mathrm{S}_{1} ; \mu_{\mathrm{T}_{1}}$ é a média das três testemunhas comerciais no teste de avaliação dos híbridos $\mathrm{S}_{1} \mathrm{xS}_{1}$; e $\mu_{\mathrm{T}_{0}}$ é a média das três testemunhas comerciais no teste de avaliação dos híbridos $\mathrm{S}_{0} \mathrm{xS}_{0}$.

\section{Resultados e Discussão}

As análises de covariância e variância dos híbridos $\mathrm{S}_{0} \mathrm{xS}_{0}$ e $\mathrm{S}_{1} \mathrm{xS}_{1}$ evidenciaram variabilidade genética, pelo teste $\mathrm{F}$, a $1 \%$ de probabilidade (Tabela 1), tanto para produtividade quanto para capacidade de expansão avaliada em pipoqueira de ar quente e na pipocadora MWVT. Isso indica que há possibilidade de se realizar melhoramento nas populações e no híbrido delas. Os coeficientes de variação experimental (CV\%) foram inferiores a $25 \%$, em relação à produtividade $\mathrm{e}$ $16 \%$ quanto à capacidade de expansão, o que revela aceitável precisão nos experimentos de campo. Esses valores são próximos aos encontrados por CarpentieriPípolo et al. (2002) e Scapim et al. (2002).

Os valores de herdabilidade, em relação à produtividade e capacidade de expansão dos híbridos 
$\mathrm{S}_{0} \mathrm{x} \mathrm{S}_{0}$ e em relação à produtividade dos híbridos $\mathrm{S}_{1} \mathrm{xS}_{1}$, são concordantes com os descritos por Pacheco et al. (1998) e Granate et al. (2002), que obtiveram valores entre 50 e $60 \%$. Quanto à capacidade de expansão, avaliada em pipoqueira de ar quente nos híbridos $\mathrm{S}_{1} \mathrm{xS}_{1}$, o valor de herdabilidade foi mais baixo, o que acarretou menores ganhos com a seleção. A redução da herdabilidade reflete diminuição da variabilidade genética, uma vez que a variação residual nos dois ensaios é praticamente a mesma $\left(7,97\right.$ e $7,79 \mathrm{~mL} \mathrm{~g}^{-1}$ para o primeiro e segundo ensaios, respectivamente). Além disso, houve uma melhor precisão experimental no segundo ensaio.

Os híbridos $\mathrm{S}_{0} \mathrm{xS}_{0}$ apresentaram produtividade satisfatória, superando inclusive a produtividade de duas das três testemunhas comerciais (Tabela 1). Esse é um aspecto positivo do ponto de vista do melhoramento, que possibilita concentração dos esforços no aumento da capacidade de expansão, principal característica de qualidade do milho-pipoca, que nos híbridos $\mathrm{S}_{0} \mathrm{xS}_{0}$ ainda está bem abaixo das testemunhas comerciais. Em relação aos híbridos $\mathrm{S}_{1} \mathrm{XS}_{1}$, tanto em relação à produtividade como para capacidade de expansão os valores foram próximos aos das testemunhas comerciais. Vendruscolo et al. (2001), testaram cultivares de milho-pipoca na região Centro-Sul do Brasil, e a cultivar com melhor desempenho apresentou produtividade média de $2.100 \mathrm{~kg} \mathrm{ha}^{-1}$ e capacidade de expansão de $21 \mathrm{~mL} \mathrm{~mL}^{-1}$ (em torno de $25 \mathrm{~mL} \mathrm{~g}^{-1}$ ). Segundo Galvão et al. (2000), para os padrões americanos, valores de capacidade de expansão acima de $25 \mathrm{~mL} \mathrm{~g}^{-1}$ são aceitáveis. No Brasil, segundo Zinsly \& Machado (1987), o valor mínimo de capacidade de expansão da população comercial deve ser $15 \mathrm{~mL} \mathrm{~g}^{-1}$.

Para os híbridos $\mathrm{S}_{0} \mathrm{xS}_{0}$, foi possível calcular a heterose, que indica a superioridade dos híbridos em relação à média dos pais (Tabela 1). Quanto à produtividade os híbridos foram superiores aos pais, com heterose de $28,3 \%$, o que demonstra haver dominância positiva para esse caráter. Souza Júnior \& Pinto (2000), trabalhando com SRR em milho, verificaram que em três ciclos de seleção a heterose para rendimento de grãos aumentou cerca de $75 \%$ a cada ciclo.

Quanto à capacidade de expansão, avaliada em pipoqueira de ar quente e em pipocadora MWVT, o valor da heterose foi positivo (3\%) e negativo (-1\%), respectivamente; no entanto, esses valores são bem próximos de zero. Esses valores de heterose tanto para produtividade quanto para capacidade de expansão são concordantes com os valores obtidos por Scapim et al. (2002), que encontraram heterose significativa para produtividade, e valores positivos e negativos não-significativos para capacidade de expansão. Segundo esses autores, isso se deveu ao

Tabela 1. Análises de covariância da produtividade $\left(\mathrm{P}, \mathrm{kg} \mathrm{ha}^{-1}\right)$ e da capacidade de expansão avaliada em pipoqueira de ar quente $\left(\mathrm{CE}_{\mathrm{aq}}, \mathrm{mL} \mathrm{g}^{-1}\right)$ e na pipocadora Metric Weight Volume Tester $\left(\mathrm{CE}_{\mathrm{MwVT}}, \mathrm{mL} \mathrm{g}^{-1}\right)$, médias dos híbridos, médias das testemunhas comerciais, herdabilidades e coeficiente de variação.

\begin{tabular}{|c|c|c|c|c|c|c|c|}
\hline \multirow[t]{3}{*}{ Fonte de variação } & & \multicolumn{6}{|c|}{ Quadrado médio } \\
\hline & \multicolumn{4}{|c|}{ Híbridos $\mathrm{S}_{0} \mathrm{x} \mathrm{S}_{0}$} & \multicolumn{3}{|c|}{ Híbridos $\mathrm{S}_{1} \mathrm{x} \mathrm{S}_{1}$} \\
\hline & GL & $\mathrm{P}$ & $\mathrm{CE}_{\mathrm{aq}}$ & $\mathrm{CE}_{\mathrm{MWVT}}$ & GL & $\mathrm{P}$ & $\mathrm{CE}_{\mathrm{aq}}$ \\
\hline Repetição & 1 & $3.597 .193,40$ & 8,22 & 8,85 & 2 & $10.805 .083,45$ & 49,02 \\
\hline Blocos/repetição & 26 & $707.009,18$ & 9,44 & 6,26 & 33 & $1.014 .946,10$ & 13,63 \\
\hline Tratamentos & 195 & $535.476,78$ & 21,27 & 18,17 & 143 & $1.732 .199,02$ & 9,76 \\
\hline Híbridos & 187 & $528.138,84 * *$ & $20,46 * *$ & $17,10 * *$ & 135 & $1.751 .686,96 * *$ & $9,44 * *$ \\
\hline Testemunhas & 7 & $726.663,83$ & 29,75 & 30,78 & 7 & $95.529 .238,04$ & 17,85 \\
\hline HxT & 1 & $971.273,15^{* *}$ & $99,59 * *$ & $159,29 * *$ & 1 & $236.006,40 * *$ & $6,64 * *$ \\
\hline Estande final & 1 & $4.063 .465,40$ & $-{ }^{(1)}$ & - & 1 & $86.400 .464,40$ & - \\
\hline Umidade & 1 & $7.556 .921,60$ & - & - & 1 & $639.873,30$ & - \\
\hline$\underline{\text { Resíduo }}$ & 169 & $268.371,62$ & 7,97 & 9,25 & 251 & $743.186,95$ & 7,79 \\
\hline Média dos híbridos & & $2.744,25$ & 17,44 & 23,30 & & $3.633,72$ & 29,25 \\
\hline Média IAC112 & & $2.974,07$ & 22,64 & 30,90 & & $3.815,39$ & 33,34 \\
\hline Média Zélia & & $1.692,53$ & 26,80 & 31,14 & & $3.430,16$ & 30,52 \\
\hline Viçosa & & $1.414,58$ & 19,40 & 26,01 & & - & - \\
\hline Beija-flor, C1 & & $2.862,69$ & 14,43 & 21,07 & & - & - \\
\hline Média Ângela & & $2.056,54$ & 24,33 & 32,14 & & $3.177,38$ & 30,84 \\
\hline Herdabilidade & & 50,37 & 60,48 & 45,69 & & 53,93 & 20,40 \\
\hline Heterose & & 605,61 & 0,525 & $-0,24$ & & - & - \\
\hline $\mathrm{CV}(\%)$ & & 20,90 & 16,09 & 12,98 & & 23,67 & 9,55 \\
\hline
\end{tabular}

${ }^{(1)}$ Dados não amostrados. **Significativo a $1 \%$ de probabilidade. 
fato de a produtividade possuir dominância positiva, enquanto a capacidade de expansão possui dominância bidirecional.

As correlações genotípicas entre produtividade e capacidade de expansão variaram de acordo com o equipamento utilizado na avaliação da última variável. Com relação aos híbridos $\mathrm{S}_{0} \mathrm{x} \mathrm{S}_{0}$, a capacidade de expansão avaliada em pipoqueira de ar quente apresentou correlação negativa com a produtividade $(-0,2949)$, concordando com os valores encontrados por Carpentieri-Pípolo et al. (2002). Valores de correlação negativos indicam que a seleção truncada em apenas um desses caracteres poderá ocasionar mudanças indesejáveis no outro. Com relação à capacidade de expansão avaliada na pipocadora MWVT, a correlação genotípica com produtividade foi positiva $(0,1640)$. A correlação entre produtividade e capacidade de expansão avaliada em pipoqueira de ar quente, para os híbridos $\mathrm{S}_{1} \mathrm{x} \mathrm{S}_{1}$, foi negativa, porém de baixa magnitude $(-0,011)$. Esse valor muito próximo de zero permite que se faça a seleção direta para capacidade de expansão, sem redução na produtividade.

Em relação aos híbridos $\mathrm{S}_{0} \mathrm{xS}_{0}$, quando se realizou seleção direta em capacidade de expansão, observaramse ganhos preditos relevantes nessa variável e ganhos negativos em produtividade (Tabela 2). Em relação aos híbridos $\mathrm{S}_{1} \mathrm{xS}_{1}$, a seleção direta em capacidade de expansão proporcionou ganho reduzido e pouco afetou a produtividade, que apresentou uma pequena redução. Isso já era esperado, pois a correlação encontrada entre as duas variáveis, embora negativa, foi de pequena magnitude, e a herdabilidade de capacidade de expansão foi reduzida. O aumento na qualidade do milho-pipoca é importante, uma vez que a seleção direta em capacidade de expansão não acarreta diminuição considerável na produtividade desses híbridos que é comparável à das populações comerciais. De acordo com Granate et al. (2002), uma alternativa para se conseguir ganhos simultâneos para CE e produtividade é por meio do uso de índices de seleção.

Foram observados ganhos realizados de $-373,79 \mathrm{~kg} \mathrm{ha}^{-1}$ e 4,83 $\mathrm{mL} \mathrm{g}^{-1}$ para produtividade e capacidade de expansão, respectivamente. O ganho realizado para capacidade de expansão avaliada na pipoqueira de ar quente foi superior ao predito $\left(2,29 \mathrm{~mL} \mathrm{~g}^{-1}\right)$. Para a produtividade, o ganho realizado também foi acima do esperado $\left(-198,93 \mathrm{~kg} \mathrm{ha}^{-1}\right)$. Rademacher et al. (1999), ao trabalhar com milho, observaram ganhos realizados para produtividade cerca de 50 a $75 \%$ menores que os ganhos preditos. O ganho para a principal característica do milho-pipoca, capacidade de expansão, teve aumentos substanciais, enquanto a redução na produtividade está dentro de limites aceitáveis, uma vez que os híbridos apresentam valores de produtividade comparável às testemunhas comerciais. Assim, com os resultados neste trabalho, o método de seleção recorrente recíproca mostrou-se satisfatório em promover melhoramento em qualidade, sem afetar de maneira significativa a produtividade, nos híbridos das populações estudadas.

A obtenção de trios completos (o híbrido e as duas famílias $\mathrm{S}_{1}$ ) na SRR é um grande obstáculo na execução dos trabalhos. Isso pode ser verificado nos números de híbridos testados $\mathrm{S}_{1} \mathrm{xS}_{1}$. Dos 136 híbridos $\mathrm{S}_{1} \mathrm{XS}_{1}$ testados, 49 tinham origem em famílias $\mathrm{S}_{1}$ de híbridos $\mathrm{S}_{0} \mathrm{xS}_{0}$ não selecionados, e apenas um tinha origem em famílias de híbridos $\mathrm{S}_{0} \mathrm{xS}_{0}$ selecionado. Os demais 86 híbridos foram formados a partir de famílias $S_{1}$ que passaram pela primeira avaliação da capacidade especifica de combinação, e algumas dessas famílias eram provenientes de híbridos $\mathrm{S}_{0} \mathrm{xS}_{0}$ selecionados e outras de não selecionados. Esse fato impede que se avalie a eficiência do processo por meio da origem dos híbridos $\mathrm{S}_{1} \mathrm{x} \mathrm{S}_{1}$ selecionados. Teoricamente, se esperaria que os híbridos $S_{1} x S_{1}$ superiores tivessem origem em famílias $\mathrm{S}_{1}$ de híbridos $\mathrm{S}_{0} \mathrm{xS}_{0}$ superiores. Entretanto, ao observar os híbridos $\mathrm{S}_{1} \mathrm{x} \mathrm{S}_{1}$ selecionados, verificou-se

Tabela 2. Ganhos preditos nas variáveis produtividade $\left(\mathrm{P}, \mathrm{kg} \mathrm{ha}^{-1}\right)$ e capacidade de expansão avaliada em pipoqueira de ar quente $\left(\mathrm{CE}_{\mathrm{aq}}, \mathrm{mL} \mathrm{g}^{-1}\right)$ e em pipocadora Metric Weight Volume Tester $\left(\mathrm{CE}_{\mathrm{MwvT}}, \mathrm{mL} \mathrm{g}^{-1}\right)$, para os híbridos $\mathrm{S}_{0} \mathrm{xS}_{0}$ e $\mathrm{S}_{1} \times \mathrm{S}_{1}{ }^{(1)}$.

\begin{tabular}{|c|c|c|c|c|c|}
\hline \multirow[t]{2}{*}{ Seleção direta } & \multicolumn{3}{|c|}{ Híbridos $\mathrm{S}_{0} \times \mathrm{SS}_{0}$} & \multicolumn{2}{|c|}{ Híbridos $\mathrm{S}_{1} \mathrm{XS}$} \\
\hline & $\mathrm{P}$ & $\mathrm{CE}_{\mathrm{aq}}$ & $\mathrm{CE}_{\mathrm{MWVT}}$ & $\mathrm{P}$ & $\mathrm{CE}_{\mathrm{aq}}$ \\
\hline Produtividade & 404,8378 & $-0,2757$ & 0,2404 & 707,8158 & $-0,1203$ \\
\hline & $(14,06)$ & $(-1,57)$ & $(1,03)$ & $(19,43)$ & $(-0,41)$ \\
\hline Capacidade de expansão (aq) & $-198,9320$ & 2,7976 & 1,5304 & $-5,4849$ & 0,4287 \\
\hline & $(-6,90)$ & $(15,94)$ & $(6,53)$ & $(-0,15)$ & $(1,47)$ \\
\hline Capacidade de expansão (MWVT) & $-55,7292$ & 1,5985 & 1,8896 & (2) & - \\
\hline
\end{tabular}

${ }^{(1)}$ Os valores entre parêntesis referem-se aos ganhos percentuais. ${ }^{(2)}$ Dados não amostrados. 
que $70 \%$ deles são de cruzamentos que passaram pelo primeiro teste de capacidade especifica de combinação. Esse fato, aliado aos substanciais ganhos realizados, indica que os híbridos $\mathrm{S}_{1} \mathrm{xS}_{1}$ são superiores aos híbridos $\mathrm{S}_{0} \mathrm{XS}_{0}$ e que sua superioridade se deveu principalmente aos efeitos de capacidade especifica de combinação. Santos et al. (2007) já haviam demonstrado em milho que os efeitos não aditivos, ou seja, de capacidade especifica de combinação são os mais explorados no melhoramento para produtividade.

Houve pouca diferença na magnitude dos valores de capacidade de expansão dos híbridos $\mathrm{S}_{1} \mathrm{xS}_{1}$ selecionados, com maior e menor valor de 32,5 e $29,41 \mathrm{~mL} \mathrm{~g}^{-1}$, respectivamente. Ao analisar as médias de capacidade de expansão dos híbridos selecionados e não selecionados, que foram de 31,36 e $29,19 \mathrm{~mL} \mathrm{~g}^{-1}$ respectivamente, pode-se perceber que a variação entre os melhores e os piores híbridos foi mínima. Portanto, a seqüência no processo de melhoramento dessas populações, visando o aumento de capacidade de expansão, pode enfrentar problemas no futuro em razão da reduzida variabilidade para essa variável. Santos et al. (2005), em três ciclos de SRR para milho, observou redução de variabilidade para todas as características avaliadas. Segundo os autores, caso a variabilidade continue a decrescer ao longo dos próximos ciclos de seleção, é indicado que novas fontes de germoplasma sejam inseridas nas populações para que seja possível a continuação do processo de melhoramento.

\section{Conclusões}

1. As populações apresentam potencial de melhoramento por si e do híbrido interpopulacional.

2. O processo de seleção recorrente recíproca é eficiente para desenvolver híbridos de famílias endógamas.

\section{Agradecimentos}

À Fundação de Amparo à Pesquisa do Estado de Minas Gerais, à Coordenação de Aperfeiçoamento de Pessoal de Nível Superior e ao Conselho Nacional de Desenvolvimento Científico e Tecnológico, pelo auxílio financeiro.

\section{Referências}

BUTRON, A.; WIDSTROM, N.W.; SNOOK, M.E.; WISEMAN, B.R. Recurrent selection for corn earworm resistance in three corn synthetics. Maydica, v.45, p.295-300, 2000.
CARPENTIERI-PÍPOLO, V.; TAKAHASHI, H.W.; ENDO, R.M.; PETEK, M.R.; SEIFERT, A.L. Correlações entre caracteres quantitativos em milho-pipoca. Horticultura Brasileira, v.20, p.551-554, 2002.

COMSTOCK, R.F.; ROBINSON, H.F.; HARVEY, P.H. A breeding procedure designed to make maximum use of both general and specific combining ability. Agronomy Journal, v.41, p.360-367, 1949.

GALVÃO, J.C.C.; SAWAZAKI, E.; MIRANDA, G.V. Comportamento de híbridos de milho-pipoca em Coimbra, Minas Gerais. Revista Ceres, v.47, p.201-218, 2000.

GRANATE, M.J.; CRUZ, C.D.; PACHECO, C.A.P. Predição de ganhos em famílias de meios irmãos do milho-pipoca CMS 43. Ciência e Agrotecnologia, v.26, p.1228-1235, 2002.

HALLAUER, A.R. Hybrid development and population improvement in maize by reciprocal full-sib selection. Egyptian Journal of Genetics and Cytology, v.2, p.84-101, 1973.

HALLAUER, A.R. Reciprocal full-sib selection in maize. Crop Science, v.24, p.755-759, 1984.

HALLAUER, A.R.; EBERHART, S.A. Reciprocal full-sib selection. Crop Science, v.10, p.315-316, 1970.

KEERATINIJAKAL, V.; LAMKEY, K.R. Responses to reciprocal recurrent selection in BSSS and BSCB1 maize populations. Crop Science, v.33, p.73-77, 1993

LITTELL, R.C.; MILLIKEN, G.A.; STROUP, W.W.; WOLFINGER, R.D. SAS System for Mixed Models. $2^{\text {nd }}$ ed. Cary: SAS Institute, 2006.

LONNQUIST, J.H.; WILLIAMS, N.E. Development of maize hybrids through selection among full-sib families. Crop Science, v.7, p.369-370, 1967.

LYNCH, M.; WALSH, B. Genetics and analysis of quantitative traits. Sunderland: Sinuauer Associates, 1998. 980p.

PACHECO, C.A.P.; GAMA, E.P.; GUIMARÃES, P.E.O.; SANTOS, M.X.; FERREIRA, A.S. Estimativas de parâmetros genéticos nas populações CMS-42 e CMS-43 de milho-pipoca. Pesquisa Agropecuária Brasileira, v.33, p.1995-2001, 1998.

PATERNIANI, E. Selection among and within half-sib families in a Brazilian population of maize (Zea mays L.). Crop Science, v.7, p.212-216, 1967.

PEREIRA, M.G.; AMARAL JÚNIOR, A.T. Estimation of genetic components in popcorn based on the nested design. Crop Breeding and Applied Biotechnology, v.1, p.3-10, 2001.

RADEMACHER, M.A.M.; HALLAUER, A.R.; RUSSELL, W.A. Comparative response of two reciprocal recurrent selection methods in BS21 and BS22 maize populations. Crop Science, v.39, p.89-97, 1999.

RUSSELL, W.A.; EBERHART, S.A. Hybrid performance of selected maize lines from reciprocal recurrent and testcross selection programs. Crop Science, v.15, p.1-4, 1975.

SANTOS, M.F.; AGUIAR, A.M.; SOUZA JÚNIOR, C.L.; MORO, G.V. Responses to a reciprocal recurrent selection and changes in genetic variability in IG-1 and IG-2 maize populations. Genetics and Molecular Biology, v.28, p.781-788, 2005. 
SANTOS, M.F.; CÂMARA, T.M.M.; MORO, G.V.; COSTA, E.F.N.; SOUZA JÚNIOR, C.L. Responses to selection and changes in combining ability after three cycles of a modified reciprocal recurrent selection in maize. Euphytica, v.157, p.185-194, 2007.

SAWAZAKI, E.; CASTRO, J.L. de; GALLO, P.B.; PATERNIANI, M.E.A.G.Z.; SILVA, R.M. da; LUDERS, R.B. Potencial de híbridos temperados de milho-pipoca em cruzamentos com o testador semitropical IAC 12. Revista Brasileira de Milho e Sorgo, v.2, p.61-70, 2003.

SCAPIM, C.A.; PACHECO, C.A.P.; TONET, A.; BRACCINI, A.L.; PINTO, R.J.B. Análise dialélica e heterose de populações de milho-pipoca. Bragantia, v.61, p.219-230, 2002.
SOUZA JÚNIOR, C.L.; PINTO, R.M.C. Responses to a short-term reciprocal recurrent selection procedure in maize. Maydica, v.45, p.21-28, 2000.

VENDRUSCOLO, E.C.G.; SCAPIN, C.A.; PACHECO, C.A.P.; OLIVEIRA, V.R. de; BRACCINI, A.L.; VIDIGAL, M.C.G. Adaptabilidade e estabilidade de cultivares de milho-pipoca na região Centro-Sul do Brasil. Pesquisa Agropecuária Brasileira, v.36, p.123-130, 2001.

ZIEGLER, K.E.; ASHMAN, B. Popcorn. In: HALLAUER, A.R. (Ed.). Specialty corns. Iowa: CRC Press, 1994. p.189-223.

ZINSLY, J.R.; MACHADO, J.A. Milho-pipoca. In: PATERNIANI, E.; VIEGAS, G.P. (Ed.). Melhoramento e Produção do Milho. 2.ed. Campinas: Fundação Cargill, 1987. v.2. p.413-421.

Recebido em 3 de julho de 2008 e aprovado em 19 de novembro de 2008 